\title{
STIMULATED ELECTRONIC RAMAN SCATTERING AS A TUNABLE INFRARED SOURCE
}

\author{
D. COTTER, D.C. HANNA, P.A. KÄRKKÄINEN, R. WYATT \\ Department of Electronics, University of Southampton, Southampton, UK
}

Received 23 May 1975

\begin{abstract}
Stimulated electronic Raman scattering in potassium vapour has been used to produce tunable infrared radiation around $2.7 \mu \mathrm{m}$. A tuning range of $\sim 1000 \mathrm{~cm}^{-1}$ has been observed with powers up to $1 \mathrm{~kW}$. The Raman output has been used to obtain an absorption spectrum of $\mathrm{CO}_{2}$ gas with a resolution of better than $0.4 \mathrm{~cm}^{-1}$.
\end{abstract}

The use of stimulated Raman scattered radiation as a tunable source has recently been reported by Schmidt and Appt [1] and Frey and Pradere [2]. By using a tunable pump source such as a dye laser the tunability can be transferred to the Raman wave, producing tunable near infrared radiation. To generate the longest possible wavelengths it is clearly desirable to work with a Raman medium having a large Stokes shift and for this reason hydrogen and methane have been used [1, 2]. However, since dyes are presently limited to wavelengths shorter than $1 \mu \mathrm{m}$ (the more efficient and reliable ones to less than $800 \mathrm{~nm}$ ) it is necessary to produce second or even third Stokes shifted radiation to reach frequencies lower than $4000 \mathrm{~cm}^{-1}$. This means that high pump powers are needed. By contrast, stimulated electronic Raman scattering $[3,4]$ can give a very large Stokes shift (e.g. $20,000 \mathrm{~cm}^{-1}$ ) and the threshold can be very low (less than $1 \mathrm{~kW}$ ) for resonance Raman scattering. Stimulated Raman scattering in potassium vapour had been used as an essential part of a tunable infrared source based on four-photon parametric scattering [5]. However, there seems to have been little interest in the Raman scattered radiation itself as a tunable source. In this letter we present some results on stimulated Raman scattering in potassium vapour which indicate that this can be a simple and useful tunable infrared source of kilowatt power with several hundred wavenumbers tuning around 2.7 $\mu \mathrm{m}$.

The relevant energy levels of potassium are shown in fig. 1. A pump laser at frequency $\nu_{\mathrm{L}}$ produces stim- ulated Raman scattering at frequency $\nu_{\mathrm{R}}$, the potassium atom being left as a result in the $5 \mathrm{~S}$ state. The power gain of the stimulated Raman scattered radiation for a length $l$ of vapour cell is $\exp (g I l)$ where the gain coefficient $g$ is given by

$$
g=\frac{N e^{4} \nu_{\mathrm{R}} f_{4 \mathrm{~S} 5 \mathrm{P}} f_{5 \mathrm{~S} 5 \mathrm{P}}}{32 \pi^{3} \epsilon_{0}^{2} h c^{2} m_{\mathrm{e}}^{2} \nu_{4 \mathrm{~S} 5 \mathrm{P}} \nu_{5 \mathrm{~S} 5 \mathrm{P}}\left(\nu_{4 \mathrm{~S} 5 \mathrm{P}}-\nu_{\mathrm{L}}\right)^{2} \Gamma},
$$

where all quantities are in MKSA units, $N$ is the density of potassium atoms, $I$ is the intensity $\left(\mathrm{W} / \mathrm{m}^{2}\right)$ of the pump laser and all frequencies including the spontaneous Raman linewidth $\Gamma$ (fwhm) are expressed in

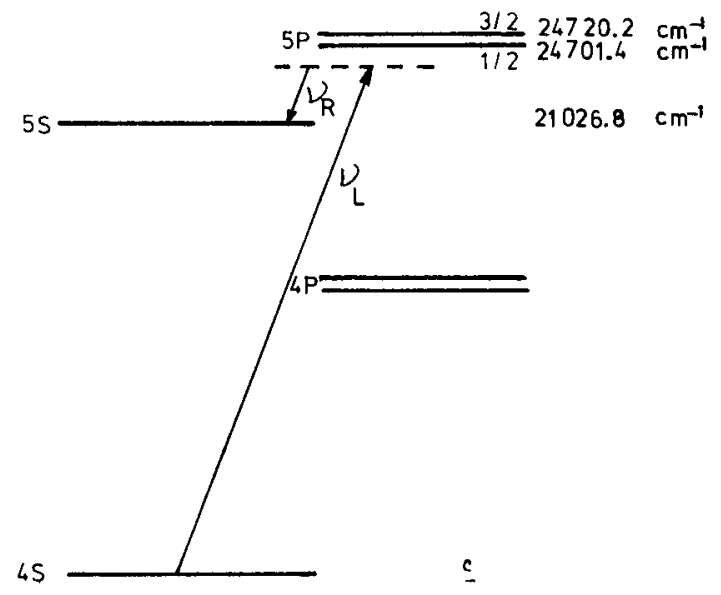

Fig. 1. Energy levels of potassium. 
Hertz. The spin - orbit splitting has been neglected and $f_{4 \mathrm{~S} 5 \mathrm{P}}, f_{5 \mathrm{~S} 5 \mathrm{P}}$ represent the total oscillator strengths for the $4 \mathrm{~S}-5 \mathrm{P}$ doublet and $5 \mathrm{~S}-5 \mathrm{P}$ doublet respectively.

Since the $5 \mathrm{P}_{3 / 2} .5 \mathrm{P}_{1 / 2}$ splitting in potassium is 18.7 $\mathrm{cm}^{-1}$, the expression given above is an adequate approximation when $\bar{\nu}_{\mathrm{L}}$ is tuned more than about 50 $\mathrm{cm}^{-1}$ off the $4 \mathrm{~S}-5 \mathrm{P}$ resonance.

The potassium vapour was contained in a doubleended, stainless steel heat pipe oven [6] of $45 \mathrm{~cm}$ length with argon buffer gas keeping the alkali vapour from the end windows. The length of the vapour column was estimated to be $30 \mathrm{~cm}$ and the vapour pressure was in the region of 10--20 torr. The pump beam was provided by a dye lāser pumped by $\sim 200 \mathrm{~kW}$ of $337 \mathrm{~nm}$ radiation from a Molectron UV-300 nitrogen laser. A solution of diphenylstilbene in p-dioxane has proved to be the most efficient dye for tuning around the $4 \mathrm{~S} 5 \mathrm{P}$ resonance $(404 \mathrm{~nm})$. This produced 20. 30 $\mathrm{kW}$ of diffraction-limited output in $\sim 0.2 \mathrm{~cm}^{-1}$ using a holographic diffraction grating combined with a prism beam-expander [8]. The dye laser beam was focused so as to be approximately confocal over the length of the vapour column.

Both forward and backward stimulated Raman scattering have been observed although most measurements have been performed on the forward scattered wave. In all of these measurements it is important to remove the various fixed frequency superfluorescent emissions. A strong emission around $1.25 \mu \mathrm{m}$, corresponding to the $5 \mathrm{~S}-4 \mathrm{P}$ transition was removed by a Ge filter. This transition occurs since the Raman pro- cess leaves potassium atoms in the $5 \mathrm{~S}$ state thus producing a population inversion between $5 \mathrm{~S}$ and $4 \mathrm{P}$ levels. In addition, emissions at 3.14 and $3.16 \mu \mathrm{m}$ were observed, corresponding to $5 \mathrm{P}-3 \mathrm{D}$ transitions. Sorokin and Lankard observed these emissions when a ruby laser pump was used [7] and suggested that they were the result of two photon absorption by $\mathrm{K}_{2}$ molecules followed by dissociation leaving $\mathrm{K}$ atoms in the $5 \mathrm{P}$ state. Like Sorokin and Lankard we have observed that the emission from the $5 \mathrm{P}$ level is on the $5 \mathrm{P}-3 \mathrm{D}$ transitions rather than the expected $5 \mathrm{P}-5 \mathrm{~S}$ transition. The Raman output energy was measured using a pyroelectric energy meter. Typically a Raman energy of $4 \mu \mathrm{J}$ was measured for a dye laser energy of $120 \mu \mathrm{J}$ into the cell. The Raman energy actually generated was therefore $\sim 10 \mu \mathrm{J}$ since the beam was attenuated by the $\mathrm{Ge}$ filter ( $\sim 40 \%$ transmission). The Raman pulse duration was not measured but it is expected to be of the same order as the dye laser pulse, duration ( $8 \mathrm{~ns}$ ), thus implying a peak Raman power somewhat in excess of 1 $\mathrm{kW}$. This power could be maintained over $\sim 250 \mathrm{~cm}^{-1}$, apart from two gaps when the pump, wavelength was within a few wavenumbers of the $4 \mathrm{~S}-5 \mathrm{P}$ doublet. Raman output was detectable (i.e. at a power level of $\sim 0.1 \mathrm{~W}$ or more) for dye laser wavelengths between 401 and $419 \mathrm{~nm}$. The corresponding Raman wavelenths are $2.56 \mu \mathrm{m}$ and $3.5 \mu \mathrm{m}$, i.e., a tuning range of $\sim 1000 \mathrm{~cm}^{-1}$. Confirmation of the Raman power measurements was provided by the observation that strong Raman generation was accompanied by almost complete depletion of the dye laser beam. The divergence

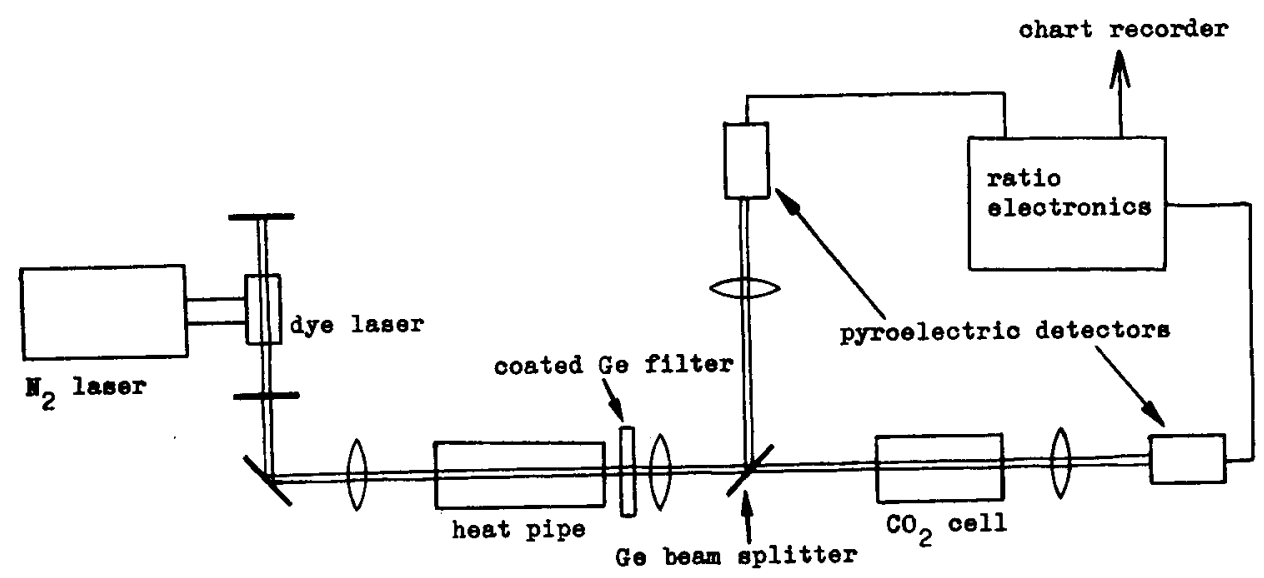

Fig. 2. Arrangement for measurement of $\mathrm{CO}_{2}$ absorption spectrum. 


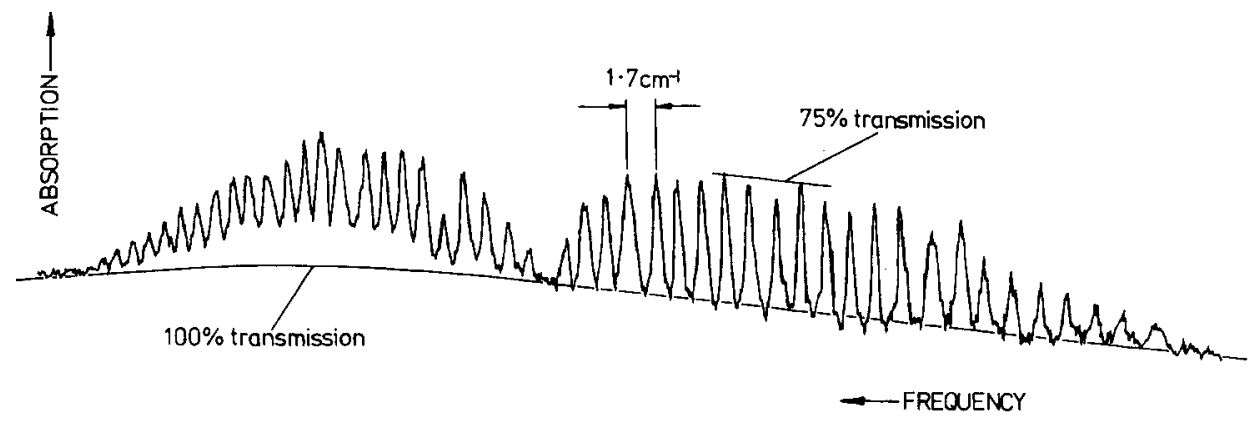

Fig. 3. Absorption spectrum of $\mathrm{CO}_{2}$ gas ( $50 \mathrm{torr}, 10 \mathrm{~cm}$ cell) around $2.7 \mu \mathrm{m}$.

of the Raman output was measured to be $25 \mathrm{mrad}$ (full angle between half intensity points) by scanning a pyroelectric detector across the beam. This is within a factor of $\sim 2$ of the calculated diffraction-limited divergence assuming the Raman beam to have the same spot size in the cell as the dye laser. This calculation neglects any gain focussing which would tend to increase the beam divergence.

Using the expression for gain given in eq. (1) and tabulated values for the oscillator strengths of potassium (see e.g. [9]) it is possible to predict the expected tuning range for a given pump power, or alternatively the threshold power for a given pump frequency. Good agreement between observed and predicted values of tuning range and threshold has been obtained at a pressure of 10 torr and with dye laser powers up to the maximum available $(\sim 10-15 \mathrm{kWatt}$ into the heat pipe). For these calculations the effective laser intensity $I$ in eq. (1) was taken to be the intensity falling within the calculated Doppler linewidth $\left(\sim 0.06 \mathrm{~cm}^{-1}\right)$. The short wavelength limit of $2.56 \mu \mathrm{m}$ was set by the limit of available dye laser tuning range. Further experiments are needed to investigate whether the effects of increasing vapour pressure, increasing laser power and narrowing the laser linewidth are in agreement with eq. (1).

To illustrate the simplicity of this technique for tunable infrared generation we have used it to produce an absorption spectrum of $\mathrm{CO}_{2}$ gas at 50 torr in a 10 $\mathrm{cm}$ length cell (fig. 2). The dye laser frequency was scanned automatically be means of a stepper-motor coupled to the micrometer drive of the grating mount. The Raman signals before and after the $\mathrm{CO}_{2}$ cell were monitored by pyroelectric detectors then ratioed and displayed on a chart recorder. Since the dye was not circulated the pulse repetition frequency was kept to $2 \mathrm{~Hz}$. The spectrum shown in fig. 3 was obtained in $\sim 50$ minutes, averaging over four pulses and stepping the dye laser by $0.05 \mathrm{~cm}^{-1}$ after every fourth pulse. Potentially the scan time can be reduced to a few minutes by incorporating dye flow and operating the nitrogen laser at its maximum repetition rate of $50 \mathrm{~Hz}$. From the spectrum it can be seen that the Raman linewidth and stability are better than $0.4 \mathrm{~cm}^{-1}$.

In conclusion, we have shown that stimulated electronic Raman scattering in potassium vapour provides a simple tunable source with several hundred wavenumbers tuning around $2.7 \mu \mathrm{m}$, with a linewidth of better than $0.4 \mathrm{~cm}^{-1}$ and powers in the kilowatt region. The short pulse duration will allow spectroscopic measurements with a time resolution of a few nanoseconds. Using more powerful dye lasers the infrared power can be readily increased and a preliminary result we have obtained using the same dye pumped by the second harmonic of a ruby laser indicates a power in excess of $10 \mathrm{~kW}$. Similar techniques applied to other vapours in particular $\mathrm{Rb}$ and $\mathrm{Cs}$ should allow extension to significantly longer infrared wavelengths [10]. In fact the number of available Raman transitions can be greatly increased by using a fixed frequency dye laser to pump atoms into an excited state and a second tunable dye laser to produce Raman scattering from that state [4].

We are grateful to Drs. T.R. Gilson and J. Black for valuable assistance.

This research has been carried out under a grant from the Paul Instrument Fund with ancillary support from the UKAEA Culham Laboratory. 


\section{References}

[1] W. Schmidt and W. Appt, Z. Naturforsch. 27a (1972) 1373;

W. Schmidt and W. Appt, Proc. VIII Intern. Quantum Electron. Conf. (1974).

[2] R. Frey and F. Pradere, Opt. Commun. 12 (1974) 98.

[3] P.P. Sorokin, N.S. Shiren, J.R. Lankard, E.C. Hammond and T.G. Kazyaka, Appl. Phys. Letters 10 (1967) 44.

[4] M. Rokni and S. Yatsiv, Phys. Letters 24A (1967) 277.

[5] P.P. Sorokin, J.J. Wynne and J.R. Lankard, Appl. Phys. Letters 22 (1973) 342;
J.J. Wynne, P.P. Sorokin and J.R. Lankard, in Proceedings of First Tunable Laser Spectroscopy Conference, Vail, Colorado, 1973 (Plenum, New York).

[6] C.R. Vidal and J. Cooper, J. Appl. Phys. 40 (1969) 3370.

[7] P.P. Sorokin and J.R. Lankard, J. Chem. Phys. 51 (1969) 2929.

[8] D.C. Hanna, P.A. Kärkkäinen and R. Wyatt, Optical and Quantum Electronics 7 (1975) 115.

[9] Atomic transition probabilities, Vol. 2, NSRDS-NBS Publication No. 22 (U.S. GPO, Washington D.C., 1969).

[10] P.P. Sorokin and J.R. Lankard, IEEE J. Quant. Electron. QE-9 (1973) 227. 